\title{
Perioperative Considerations in Rheumatoid Arthritis Patients
}

\author{
Derar Seyoum, Wasim Khan
}

UCL Institute of Orthopaedics and Musculoskeletal Sciences, Royal National
Orthopaedic Hospital, Stanmore, HA7 4LP, UK

\author{
Corresponding Author: Mr WS Khan, Clinical Lecturer, University College \\ London Institute of Orthopaedics and Musculoskeletal Science, Royal National \\ Orthopaedic Hospital, Stanmore, Middlesex, London, HA7 4LP, UK \\ Telephone number: +44 (0) 7791025554 \\ E-mail address: wasimkhan@doctors.org.uk
}

\begin{abstract}
The pre-operative assessment is used to clarify issues prior to surgery that can change management provided by a multidisciplinary team. A high proportion of rheumatoid arthritis patients are elderly requiring orthopaedic surgery which requires further investigations, assessment of risk through functional capacity and several anaesthetic considerations. Rheumatoid arthritis patients often provide a variety of medical issues that can be found on careful and thorough pre-assessment that can be accommodated for by the surgical and anaesthetic team, however aspects of holistic management is an important considerations for efficient and effective management.
\end{abstract}

\section{$\underline{\text { Introduction }}$}

The purpose of the pre-operative assessment is to identify any known or formerly unknown comorbidities that may have an impact on anaesthetic and surgical decisions, with this information and an understanding the patients baseline status, the patient can be risk stratified in a manner to identify any potential peri-operative and post-operative complications of surgery [1]. With the aging UK population there are a growing number of patients with rheumatological conditions which progress on to a greater surgical demand on surgery for rheumatoid arthritis patients. In a report by the NCEPOD 1999 reviewing deaths in elderly patients, $60 \%$ of patients were under the care of orthopaedic surgeons at the time of their final surgery[2]. In this review, we assess the pre-operative assessment taking into considerations medical considerations of rheumatoid arthritis patients and value of investigations and multidisciplinary team issues.

\section{General Medical Considerations}

Like all patients rheumatoid arthritis patients have to have baseline assessment to identify if they are fit for surgery. Therefore all patients should have oxygen saturations; temperature, blood pressure and resting pulse are kept in record. Though adapted when necessary further tests are done especially if they are of certain patient group. ECGs are done for patients with diabetes, a history of hypertension, heart disease or if they are over 50 years old. A full blood count is done for patients with a history of chronic rheumatoid arthritis. Clotting profiles are done for patient on anticoagulants or have a history of bruising or bleeding. Urea and electrolytes are useful if patients 
are on immunosuppressive therapy or diuretics. Patients known to have an advanced respiratory disease or who have a low pulse oximetry would have an arterial blood gas. Also for those patients who have limiting pulmonary symptoms a vitalograph would be used. More formal guidance is offered on the NICE guidelines [3].

From an anaesthetists perspective, the Association of Anaesthetists of Great Britain and Ireland (AAGBI) has shown that independent prognostic information regarding perioperative risk has to be determined by nine specific factors [4]. Patients who have ischaemic heart disease (IHD), heart failure, ischaemic brain disease, kidney failure and peripheral arterial disease factors in the medical history provide prognostic information. The other four factors are age, gender, socioeconomic status and aerobic fitness can act as perioperative risk predictors. The physical activity and environmental factors also are valuable in identifying perioperative risk. Paying attention to chronic medical conditions together with the respect for how co-morbidities can combine to have a multisystem effect helps in providing a more consistent approach to pre-operative risk assessment [4].

\section{Cardiovascular Risk}

The American Heart Association (AHA) and the American College of Cardiology recommend that four cardiac conditions require further evaluation and investigation, and optimization [4].

Decompensated heart failure with a New York Heart Association Class IV, Unstable Coronary Syndrome, significant arrhythmias and severe valve disease if identified are the areas for attention. The AHA and ACC suggest identifying patients who are at risk of cardiovascular disease by looking for predisposing factors for example a history of myocardial infarction, prior angina, existing heart failure, presence of a pacemaker or implantable cardioverter defibrillator or history of orthostatic intolerance. Note any indications of related disease like renal impairment or chronic pulmonary disease; document any recent changes in symptoms in cardiac patients, and all their medications. If the patient has a pacemaker, identify the reason for the pacemaker and the patients underlying rhythm. Also it is important to determine the patient's functional capacity [4].

For cardiac risk the investigation often of choice is the exercise tolerance test where patients on a treadmill are closely monitored through increasing workloads however certain rheumatoid patient may be unable to use a treadmill so arm-cycle ergometry would be used as an alternative. If the patient is unable to do the exercise test Dobutamine stress echocardiography or Thallium scintigraphy can be used as another alternative [2].

For patients with an American Society of Anaesthesiologists physical status score of 3 or more, a full blood count and renal function tests are done. For identifying heart failure, cardiac biomarkers such as N-terminal pro-brain natriuretic peptide (NT-pro-BNP) may be a more helpful screening tool compared to echocardiography, and when positive more information can be gathered with dynamic tests like Dobutamine stress test [4]. However echocardiography is indicated in major structural abnormalities such as aortic stenosis, though dynamic testing can identify the significance of such abnormalities and whether they require valve replacement or prophylactic antibiotics. Underlying rhythm disturbances would need to be corrected with a pacemaker if are of second or third-degree block [2].

\section{$\underline{\text { Respiratory Risk }}$}

Respiratory complications such as infection, exacerbation of chronic disease, atelectasis and bronchospasm are common after surgery. So there are important factors to assess for the risk of pulmonary complications as recommended by the American College of Physicians are conditions such as chronic obstructive pulmonary disease, congestive heart failure, and in terms of patient 
status if they are 60 years and above, have an ASA class 2 or greater, and a low albumin [4]. However NICE views that most respirator disease investigations are non-prognostic and that pulmonary function test, Chest X-rays and arterial blood gas analysis have doubtful value. But baseline investigations have validity [4].

In terms of respiratory conditions that can be easily missed, obstructive sleeps apnoea is undiagnosed in 80 to $90 \%$ of OSA patients [5]. Complications that can develop as a consequence of OSA are airway obstruction, cardiac events, difficulty intubating and postoperative respiratory depression. So patients who are discharged with undiagnosed OSA are at risk of complications especially due to opioid pain management which is very relevant to rheumatoid arthritis patients [6].

A controversial consideration is obesity. Patients who are obese have a propensity for intraoperative respiratory events and often have underlying conditions like restrictive lung disease, OSA, hypoxemia, hypercapnia and chronic obstructive lung disease [7]. However Miguel [8] identified that obesity is not a risk factor for postoperative pulmonary complications. Another patient factor is smoking patients who are at an increased risk of post-operative pulmonary complications even if there is no chronic lung disease [8].

As mentioned surgical considerations are important to assess for risk of pulmonary complications. The site of the surgery has been shown to have an effect on risk of post-operative respiratory complications. The distance of the incision to the patient diaphragm is inversely related to the rate of complications developed [8]. Also an increased length of surgery beyond 3-4 hours can increase the risk of pulmonary complications. In terms of anaesthesia, spinal or epidural anaesthesia has a reduced risk of respiratory complications relative to general anaesthesia [8].

\section{$\underline{\text { Further Medical Risks }}$}

Renal failure is an important independent perioperative risk factor for mortality and morbidity. It has been reported that acute kidney injury that occurs preoperatively has an 8 times increase risk of mortality [4]. Risk factors for acute kidney injury occurring are mild and moderate renal insufficiency, hypertension, active heart failure and diabetes mellitus. NICE indicate that investigations for renal impairment should be carried out for patients with known kidney disease, patients with diabetes and hypertension, patients undergoing surgery of a surgical severity score 4, patients undergoing surgery of a surgical severity of 3 whom are over 60 years old, and all patients with an ASA grade 3 due to cardiovascular disease [5].

As rheumatoid conditions are more prevalent in the elderly population, neurological issues such as confusion can be identified in pre-operative assessments and conditions such as dementia should be diagnosed after expert review [2]. Thus it is important reversible causes of confusion such as infection, hypoxia and electrolyte disturbances. Neurological impairment like dementia and confusion can be very difficult to assess thus the importance of collateral histories, and also it should be pointed out that post-operative symptoms may be worse [2].

\section{Rheumatoid Arthritis Specific Considerations}

\section{Cervical Spine}

A very important part of the pre-operative assessment of rheumatoid arthritis patients is the cervical spine $x$-ray to identify cervical subluxation. The cervical spine is the $2^{\text {nd }}$ most common area of destruction in rheumatoid arthritis after the metacarpophalangeal joint [9]. It has been shown that cord compression accounts for $10 \%$ of deaths in patients with rheumatoid cervical spine 
involvement. In $43 \pm 86 \%$ of rheumatoid arthritis patients have been found to have cervical subluxation and the severity is related to the duration of rheumatoid disease [9]. Cervical subluxation is commoner in patients with high rheumatoid factor levels, severe peripheral arthritis and nodules. However cervical subluxation due to rheumatoid arthritis is asymptomatic in $50 \%$ and it is difficult to detect through neurological deficits when patients have arthritic joints and weakness and atrophy related to rheumatoid arthritis [9].

The commonest type of cervical subluxation is anterior atlanto-axial subluxation which makes up for $50 \pm 70 \%$ of all cervical subluxations in rheumatoid arthritis patients, related to transverse ligament damage or instability [9]. The second most common is Sub axial subluxation of the lower cervical vertebrae that makes up for $20 \pm 25 \%$ [9]. Sub axial subluxation is arthritis of the facet joints, disc involvement and laxity of the ligaments and can produce a 'step-ladder' deformity. Thirdly and most dangerous form of cervical subluxation is Basilar Invagination accounting for $10 \pm 15 \%$, this is also known as vertical atlanto-axial subluxation, atlanto-axial impaction or superior migration of the odontoid [9]. The rare other forms are posterior and rotatory atlanto-axial subluxations. If such lesions are identified discussion with anaesthetists and surgeons is required and soft cervical collar or surgical support should be used to immobilize and prevent over manipulation of the neck.

Cervical subluxation is detected with the use of lateral cervical spine radiographs taken in flexion and extension. However prior to this neck movements should be performed to clarify the range of movements and the patient should be asked if any movements cause pain and stiffness can limit the range of movement. To identify atlanto-axial subluxation (AAS) the anterior atlanto-dental interval should be greater than $3 \mathrm{~mm}$ in adults; it is the measurement from the anterior surface of the odontoid to the posterior inferior aspect of the anterior arch of the atlas. Sub axial subluxation is a translation of one of the vertebral body on another by greater than $3.5 \mathrm{~mm}$ [9]. The SAS relates to the sagittal diameter of the spinal canal and should be at least $14 \mathrm{~mm}$ all the way through and be a good predictor of the development of paralysis. Basilar Invagination is often unrecognized and can also coexist with AAS. Traditionally it has been identified using McGregor's Method which is an odontoid protrusion of greater than $4.5 \mathrm{~mm}$ above McGregor's line (a line drawn from most caudal part of the occiput to the postero-superior aspect of the hard palate) [9]. However it can be difficult to apply thus other methods such as Runaway, Redlund-Johnell and Kauppi Method are used [9].

\section{Systemic manifestations of Rheumatoid Disease}

Rheumatoid arthritis also has non-articular manifestations through different systems. In the lung it can lead to diffuse fibrosing alveolitis that can produce a restrictive lung picture on lung function tests. Cardiac manifestations are pericarditis which is asymptomatic in $45 \%$ but can lead to large pericardial effusions [2]. Rheumatoid arthritis patients can have renal involvement from amyloid, interstitial nephritis or autoimmune Nephropathy. Amyloidosis can lead to nephrotic syndrome and renal failure and can present with proteinuria [2]. Rheumatoid arthritis patients can also develop anaemia typically normochromic normocytic but could also be iron deficient. Neuropathies can be a non-articular manifestation of rheumatoid disease, glove and stocking sensory loss due to vasculitis [2].

\section{$\underline{\text { Medications and anaesthesia }}$}

Every medication the patient taking should be documented in the pre-operative assessment including illicit drugs, herbal remedies and over-the-counter medications allowing the surgical and anaesthetic team to assess whether a patient is adequate for surgery and if any interactions have to be considered [1]. Typically cardiac or blood pressure medications are taken however discussion with anaesthetist in coordinating ideal pre-operative medications [10]. However patients on 
angiotensin-converting enzyme inhibitors are more prone to circulatory instability [10] and diuretics may be omitted and careful oral fluid intake monitored to prevent dehydration or large fluid volume shifts or fluid overload, which elderly patients have an impaired ability to cope with [2].

Drugs used to treat the disease predispose patients to adverse effects. Patients on chronic corticosteroid therapy may require prophylaxis from adrenal insufficiency for the stress of surgery using a bolus dose of intravenous hydrocortisone [10]. Peptic ulcer disease is common in rheumatoid arthritis patients and can also occur as a consequence of use of corticosteroids, non-steroid antiinflammatory drugs and exacerbated peri-operatively and is difficult to manage if the patient is placed on anticoagulants or on indomethacin for heterotopic ossification [10]. Therefore an active peptic ulcer should be identified and managed effectively prior to proceeding with surgery. Patients with a history of gastrointestinal bleeding, peptic ulcer disease or active symptoms of dyspepsia should receive prophylactic $\mathrm{H} 2$ blocker therapy[10].

In terms of analgesia patients whom oral narcotics are part of their regular medication preoperatively may require a higher narcotic requirement and may have difficulty with pain control post-operatively [10]. Patient -controlled analgesia is an effective form of post-operative pain control however rheumatoid arthritis patients with significant hand deformities may find their use impractical [10].

For anaesthetic it is the choice of the anaesthetist for which techniques and agents are selected dependent on the specific needs of the patient and the type of procedure being carried out. For rheumatoid arthritis patients who may have temporomandibular joint involvement thus restricting jaw opening and patients with arthritis in the cricoarytenoid joints the anaesthetist should be informed as this may affect the Mallampati score and choice of airway management. For patients who have a rigid airway or cervical spine instability the use of fibreoptic intubation may be needed [11].

For consideration of anaesthetic agents used, based on the medical history any hepatic or renal impairment may require anaesthetic agents that are metabolised through alternative means such as via plasma esterases [11]. Cardiac pathology may require the consideration of medication that does causes less of an effect on the cardio-respiratory system. In terms of anaesthesia, spinal or epidural anaesthesia has been shown to have a reduced risk of transfusion requirement, incidence of pneumonia, respiratory depression and reduced mortality relative to general anaesthesia [12]. Whilst there is the benefit that general, inhalatory anaesthesia allows more control of the respiratory and cardiovascular systems thus not limiting the time of surgery [10]. Epidural anaesthesia reduces the chance of venous thromboembolism relative to general anaesthesia and reduces intraoperative blood loss [8].

\section{Rheumatoid medications}

Nonsteroidal Anti-Inflammatory Drugs (NSAIDs) are often used in patients with rheumatoid arthritis. In view of their effect on platelet aggregation and bleeding time, we recommend discontinuing NSAIDs with long half-life one week before surgery, and short half-life drugs 2-3 days before surgery.

Almost $80 \%$ of rheumatoid arthritis patients take drug therapy for their condition including Disease Modifying Anti-Rheumatic Medication (DMARDs) and these need special consideration in the perioperative period [13]. It is important to ensure good communication between the managing rheumatologist and the operating surgeon. The complication rate is higher in rheumatoid arthritis patients, particularly for infections. Traditional DMARDs e.g. methotrexate, hydroxychloroquine, sulfasalazine and azathioprine are safe in the peri-operative period, but it is important to ensure the 
renal function is monitored. There is a lack of clinical data for tofacitinib, but higher infection rates have been noted, and recent publications recommend withholding it for 2 days [13-15].

Pooled data suggests that anti-TNF $\alpha$ inhibitor therapy significantly increases the risk of surgical site infection [14]. Some drugs e.g. tocilizumab may not increase the risk of peri-operative infection, but may mask the clinical signs of infection making it more difficult to recognise. As there is insufficient data on abatacept, it should be stopped for at least 2 weeks pre-operatively. The risk of perioperative infection with rituximab is not related to the interval between drug administration and surgery, but some authors have suggested measuring immunoglobulin levels in high-risk patients [14].

As there is little data regarding the optimal time to stop these medications pre-operatively and to start them post-operatively, most authors recommend stopping them for 1.5 times the dosing interval, and starting them once the wound has healed i.e. two weeks post-operatively $[13,14]$. The pre-operative duration that TNF $\alpha$ inhibitors need to be stopped works out to be 2 weeks for etanercept, 3 weeks for adalimumab, and 6 weeks for golimumab, infliximab and certolizumab.

\section{Multidisciplinary team aspect of Preoperative Assessment}

Components for the success of a team in pre-operative assessment are the core values which underlie practice. Lucas [16] points out that commitment to the patients, to the professional development of staff, commitment to the team and the trust contribute to the development of an effective patient centred service. Lowry [17] also establishes that monthly multidisciplinary team meetings and collaboration with the primary care teams to develop contact with patient are important.

Lowry [17] looked at different methods of pre-assessment work up and identified three systems. The first system being the traditional format where pre-assessment uncovers problems thus preventing admission and requiring a replacement on short notice. The second system 'Revise Procedure' provides a date for the surgery once the patient has had all tests done and documented and deemed fit for surgery. As problems are dealt with prior to dating of surgery it has a low cancelation rate. The third system 'One-stop shop' is where the nurse organises appointments to the pre-assessment clinic and sees the patient ordering all the investigations. The consultant then sees the patient and takes consent, an on-call occupational therapist is available to deal with any problems identified by the nurse, and then finally the anaesthetist sees the patients. When all the professionals are satisfied and the patient is happy, a date for surgery can be arranged. As well as given a very low problem rate, patient satisfaction rate is higher. Because the nurses the patient they are the patients point of contact for any problems.

The nurses role are key for assessment and patient education, which can expand to a more full holistic patients assessment and can concentrate on problems that may cause delay in discharge, such as clinical co-ordination and social circumstances [16]. Rates of cancellation and patient visits to emergency services after discharge and rates of transfers and admissions can act as quality metrics for multidisciplinary teams to assess by for need any needs in improvement [1].

\section{Conclusion}

A thorough pre-operative assessment can be carried out with recording a detailed clinical history, a screening pre-operative questionnaire, clinical examination and routine test with necessary further individualised investigations, risk-assessments and fasting policies based on guidelines. See Table 1 where we have produced a key considerations check list for rheumatoid arthritis patient undergoing 
surgery. Other aspects that are taken into consideration are costs of routine test, the legal responsibilities of the medical team and the patient's perspective. The act of systematically carrying out the pre-operative assessment put focus on variations of tests and management plans of rheumatoid arthritis patients.

The development pre-operative assessments of rheumatoid arthritis patients helps to effectively individualise pre-operative assessments and accommodate for rheumatoid arthritis patients in surgery and out of surgery with a means of an effective and supportive patient pathway taking into consideration the patient's views and availability, is vital for managing the growing demand of growing surgical requirement for rheumatoid arthritispatients

\section{$\underline{\text { References }}$}

1. Allison J, George M. Using Preoperative Assessment and Patient Instruction to Improve Patient Safety. AORN Journal 2014; 99(3): 364-75.

2. Hudson P, Shinner G. Preoperative assessment of the orthopaedic patient. Anaesth \& Int Care Med 2006; 7(3): 72-5.

3. National Collaborating Centre for Acute Care (NCCAC) Preoperative tests, the use of routine preoperative tests for elective surgery- evidence, methods and guidance. London: NICE, 2003. http://www. nice.org.uk/pdf/Preop_Fullguideline.pdf

4. Burnside WS, Snowden C, Preoperative assessment and investigation. Surgery 2014; 32(2): 63-8.

5. Patients and consumers. Do you have obstructive sleep apnea? Patient Safety Authority. http://patientsafety authority.org/PATIENTSCONSUMERS/Patient ConsumerTips/Pages/OSA_Consumer_Tips.aspx. Published September 2007. Accessed November 25, 2013.

6. Gross JB, Bachenberg KL, Benumof JL, et al. American Society of Anesthesiologists Task Force on Perioperative Management of Patients with Obstructive Sleep Apnea. Practice guidelines for the perioperative management of patients with obstructive sleep apnea: report by the American Society of Anesthesiologists Task Force on Perioperative Management of Patients with Obstructive Sleep Apnea. Anesthesiol 2006; 104(5): 108193.

7. Adams JP, Murphy PG. Obesity in anaesthesia and intensive care. Br J Anaesth 2000; 85(1): 91-108.

8. Garcia-Miguel FJ, Serrano-Aguilar PG, Lopez-Bastida J. Preoperative assessment. Lancet 2003; 362: 1749-57.

9. Roche CJ, Eyes BE, Whitehouse GH. The Rheumatoid Cervical Spine: Signs of Instability on Plain Cervical Radiographs. Clin Radiol 2002; 57: 241-9.

10. Mackenzie CR, Sharrock NE. Perioperative Medical Considerations in Patients with rheumatoid Arthritis. Rheum Dis Clin North Am 1998; 24(1): 1-17.

11. Aires RB, de Carvalho JF, da Mota LCH. Pre-operative anesthetic assessment of patients with rheumatoid arthritis. Rev Bras Reumatol 2014; 54(3): 213-9.

12. Rodgers A, Walker N, Schug S, et al. Reduction of postoperative mortality and morbidity with epidural or spinal anaesthesia: results from overview of randomised trials. BMJ 2000; 321: 1493-96.

13. Krause ML, Matteson EL. Perioperative management of the patient with rheumatoid arthritis. World J Orthop 2014; 5(3): 283-91.

14. Goodman SM. Rheumatoid arthritis: Perioperative management of biologics and DMARDs. Semin Arthritis Rheum 2015; 44(6): 627-32.

15. Goodman SM. Optimizing perioperative outcomes for older patients with rheumatoid arthritis undergoing arthroplasty: emphasis on medication management. Drugs Aging 2015; 32(5): 361-9. 
16. Lucas B. Developing the role of the nurse in the orthopaedic outpatient and pre admission assessment settings: a change management project. J Orthop Nurs 2002; 10(6): 153-60.

17. Lowry L, Lewis V. Redesigning an orthopaedic pre-assessment clinic. J Orthop Nurs 2004; 8: 77-82. 


\begin{tabular}{|l|l|}
\hline \multicolumn{2}{|l|}{ Key Considerations Check list for Rheumatoid Arthritis Patient Undergoing Surgery } \\
\hline $\begin{array}{l}\text { Baseline observations including oxygen saturations, temperature, blood pressure } \\
\text { and resting pulse }\end{array}$ & \\
\hline $\begin{array}{l}\text { Additional investigations if indicated including ECG, blood tests (FBC, clotting } \\
\text { profile, and urea and electrolytes) }\end{array}$ & \\
\hline $\begin{array}{l}\text { Anaesthetic assessment including airway consideration, cervical spine assessment } \\
\text { and choice of anaesthetic drugs }\end{array}$ & \\
\hline $\begin{array}{l}\text { Cardiac investigations: Exercise tolerance test or alternatively Dobutamine stress } \\
\text { echocardiography or Thallium scintigraphy, and cardiac biomarkers such as N- } \\
\text { terminal pro-brain natriuretic peptide (NT-pro-BNP) or echocardiography }\end{array}$ & $\begin{array}{l}\text { Respiratory investigations: Arterial blood gas, vitalograph, pulmonary function test } \\
\text { and chest X-rays }\end{array}$ \\
\hline $\begin{array}{l}\text { DMARDs sopped and started according to recommendations and following } \\
\text { discussion with rheumatologists. Ensure adequate analgesia peri-operatively }\end{array}$ & \\
\hline Multidisciplinary team assessment & \\
\hline
\end{tabular}

Table 1: Key Considerations Check list for Rheumatoid Arthritis Patient Undergoing Surgery 